\title{
When students become patients: TB disease among medical undergraduates in Cape Town, South Africa
}

\author{
H van der Westhuizen, ${ }^{1,2}$ MB ChB; A Dramowski, ${ }^{2,3}$ MB ChB, PhD, FCPaed, MMed (Paed), Cert Paed ID, DCH \\ ${ }^{1}$ Department of Global Health, Division of Health Systems and Public Health, Faculty of Medicine and Health Sciences, Stellenbosch University, \\ Cape Town, South Africa \\ ${ }^{2}$ TB Proof, Cape Town, South Africa \\ ${ }^{3}$ Department of Paediatrics and Child Health, Division of Paediatric Infectious Diseases, Faculty of Medicine and Health Sciences, Stellenbosch \\ University, Cape Town, South Africa
}

Corresponding author: H van der Westhuizen (helene1mari@gmail.com)

\begin{abstract}
Background. Medical students acquire latent tuberculosis (TB) infection at a rate of 23 cases/100 person-years. The frequency and impact of occupational TB disease in this population are unknown.

Methods. A self-administered questionnaire was distributed via email and social media to current medical students and recently graduated doctors (2010 - 2015) at two medical schools in Cape Town. Individuals who had developed TB disease as undergraduate students were eligible to participate. Quantitative and qualitative data collected from the questionnaire and semi-structured interviews were analysed with descriptive statistics and a framework approach to identify emerging themes.

Results. Twelve individuals (10 female) reported a diagnosis of TB: pulmonary TB ( $n=6)$, pleural TB $(n=3)$, TB lymphadenitis $(n=2)$ and TB spine ( $n=1) ; 2 / 12$ (17\%) had drug-resistant disease (DR-TB). Mean diagnostic delay post consultation was 8.1 weeks, with only $42 \%$ of initial diagnoses being correct. Most consulted private healthcare providers (general practitioners $(n=7)$; pulmonologists $(n=4)$ ), and nine underwent invasive procedures (bronchoscopy, pleural fluid aspiration and tissue biopsy). Substantial healthcare costs were incurred (mean ZAR25 000 for drug-sensitive TB, up to ZAR104 000 for DR-TB). Students struggled to obtain treatment, incurred high transport costs and missed academic time. Students with DR-TB interrupted their studies and experienced severe side-effects (hepatotoxicity, depression and permanent ototoxicity). Most participants cited poor TB infection-control practices at their training hospitals as a major risk factor for occupational TB.

Conclusions. Undergraduate medical students in Cape Town are at high risk of occupationally acquired TB, with an unmet need for comprehensive occupational health services and support.
\end{abstract}

S Afr Med J 2017;107(6):475-479. DOI:10.7196/SAMJ.2017.v107i6.12260

Medical students in South Africa (SA) complete undergraduate training in communities with extremely high tuberculosis (TB) incidence rates (834 per 100000 population in 2015). ${ }^{[1]} \mathrm{SA}$ healthcare workers (HCWs) are at high risk of developing TB: three times increased risk for drug-sensitive TB (DS-TB) and up to 6.7 times increased risk for drug-resistant TB (DR-TB) when compared with the general population. ${ }^{[2-4]}$ Poor implementation of TB-infection control measures (TB-IC) is reported from SA healthcare facilites, contributing to TB exposure and development of occupational TB disease among SA HCWs and health science students. ${ }^{[5,6]}$

Latent TB incidence among SA medical students was measured by Tuberculin Skin Test (TST) conversion at 23 cases/100 personyears $(95 \%$ confidence interval (CI) $12-43) .{ }^{[7]}$ While this was lower than that of SA HCWs, this rate is three times higher than the average annual risk of TST conversion in HCWs from five other high-TB-burden countries (8.4/100; 95\% CI 2.7 - 14) ${ }^{[8]}$ Knowledge about TB-IC has been shown to reduce the odds of a positive TST in medical students by $>70 \%$ (adjusted odds ratio (OR) $0.29 ; 95 \%$ CI 0.09 - 0.98), indicating the importance of TB-IC training at undergraduate level. ${ }^{[9]}$ However, even if TB-IC knowledge can be improved at undergraduate level, the lack of clinician role models may impact TB-IC practices negatively. ${ }^{[10]}$
In a study reporting SA doctors' experiences with occupational TB disease, significant diagnostic delays and high rates of invasive procedures were documented. ${ }^{[1]}$ In addition to high mortality, morbidity and treatment side-effects, DR-TB has been demonstrated to have profound psychosocial impact among SA HCWs. ${ }^{[12,13]}$ Although data on the incidence and impact of occupational TB in SA HCWs are sparse, even less is known about the experiences of SA medical students with occupational TB. We aimed to: (i) investigate the clinical presentation, diagnostic investigations and treatment of TB in medical students; (ii) describe students' experiences of developing occupational TB; and (iii) evaluate the support systems utilised following TB diagnosis.

\section{Methods}

This descriptive cross-sectional study was conducted in 2015. Medical students at two medical training universities in the Western Cape Province, SA, who had developed TB during their undergraduate medical training between 2010 and 2015, were eligible to complete an online questionnaire. Those who developed TB after graduation or prior to the commencement of their studies were not eligible for inclusion. A total of 3500 individuals was approached to participate in the study: 2028 (58\%) were current medical students in their clinical training years at Stellenbosch University and the University 
of Cape Town who received notification of the study via university mailing lists, and 1472 (42\%) were recent medical graduates from the two universities $(2010$ - 2015) who were notified via email and social media (Facebook groups and the TB Proof Facebook page). A lucky-draw cash prize was offered as an incentive for participation. All students who met the inclusion criteria were eligible to complete a self-administered electronic questionnaire that contained 15 quantitative and 14 qualitative questions. The questionnaire was developed in consultation with occupational health and infection control experts, and was piloted and reviewed to ensure it contained no stigmatising language.

A subset of the questionnaire respondents was approached to participate in 30-minute semi-structured individual interviews (two participants from each university were selected based on their ability to attend the interview). Interviews were transcribed by a trained senior researcher and coded by both investigators using a master code list. Differences in coding were resolved through discussion between the two coders. A framework approach was used to identify emerging themes. Both quantitative and qualitative responses were grouped into four domains: (i) utilisation of healthcare services; (ii) impact of disease; (iii) support structures; and (iv) TB infection control in training hospitals. Qualitative data describing the impact of stigma will be reported in a separate article. Participant number (P) is indicated before the selected quotations. Quantitative data were analysed using frequencies and percentages and reported using mean with standard deviation (SD).

Ethical approval was obtained from the Health Research Ethics Committees of Stellenbosch University (ref. no. S15/02/025) and the University of Cape Town (ref. no. 331/2015). Institutional permission was obtained from both universities.

\section{Results}

\section{Demographics and disease presentation}

Twelve individuals (mean age 24 years) self-reported a diagnosis of TB disease during their undergraduate training years. Table 1 illustrates demographic information and summarises TB disease presentation.

\section{Utilisation of healthcare services}

The majority of participants utilised private healthcare providers, specifically general practitioners $(n=7)$ and pulmonologists $(n=4)$. Despite some students visiting the universities' campus health services $(n=3)$, diagnostic investigations were not available, and students were referred to private practitioners or public tertiary training hospitals. Participants experienced significant delays in TB diagnosis: the mean delay between developing symptoms and seeking help was 3.2 weeks (range 0 - 16 weeks, SD 4.6); the mean diagnostic delay post consultation was 8.1 weeks (range 0 - 32 weeks, SD 12.3). Fear of missing academic teaching and clinical duties influenced students' health-seeking behaviour and delayed TB diagnosis:

'Medical school trains you to believe that school is more important and that health comes second. There is no time to go to a doctor and I stayed with that lymph node for 2 weeks and it got bigger and more painful. [It is ironic that] our problem is we don't have time to go to the clinic and doctors are roaming our corridors.' (P2)

Health-system delays were the biggest contributing factor to diagnostic delays, including difficulty accessing services during holidays, long waiting times at public hospitals and a low index of suspicion among private healthcare providers. Only $41.7 \%$ of initial diagnoses were correct, with misdiagnoses ranging from pneumonia $(n=3)$ to uncontrolled asthma $(n=1)$, influenza $(n=1)$,
Table 1. Demographic information $(N=12)$

\begin{tabular}{|c|c|}
\hline & $n(\%)$ \\
\hline \multicolumn{2}{|l|}{ Gender } \\
\hline Female & $10(83.3)$ \\
\hline Male & $2(16.7)$ \\
\hline \multicolumn{2}{|l|}{ Age (years) } \\
\hline $21-23$ & $4(33.3)$ \\
\hline $24-26$ & $8(66.6)$ \\
\hline \multicolumn{2}{|l|}{ Year of study at TB diagnosis } \\
\hline 2nd & $2(16.7)$ \\
\hline $3 \mathrm{rd}$ & $1(8.3)$ \\
\hline 4 th & $6(50.0)$ \\
\hline 5 th & $1(8.3)$ \\
\hline 6th & $2(16.7)$ \\
\hline \multicolumn{2}{|l|}{ Period during which TB diagnosis was made } \\
\hline $2010-2012$ & $5(41.7)$ \\
\hline $2013-2015$ & $7(58.3)$ \\
\hline \multicolumn{2}{|l|}{ Training institution } \\
\hline Stellenbosch University & $7(58.3)$ \\
\hline University of Cape Town & $5(41.7)$ \\
\hline \multicolumn{2}{|l|}{ Site of TB } \\
\hline Pulmonary TB & $6(50.0)$ \\
\hline Pleural TB & $3(25.0)$ \\
\hline TB lymphadenitis & $2(16.7)$ \\
\hline TB spine & $1(8.3)$ \\
\hline \multicolumn{2}{|l|}{ Drug sensitivity } \\
\hline Drug-sensitive TB (DS-TB) & $10(83.3)$ \\
\hline Drug-resistant TB (DR-TB) & $2(16.7)$ \\
\hline \multicolumn{2}{|l|}{ Baseline presenting symptoms* } \\
\hline Malaise & $7(58.3)$ \\
\hline Cough & $7(58.3)$ \\
\hline Weight loss & $5(41.7)$ \\
\hline Night sweats & $4(33.3)$ \\
\hline Fever & $3(25.0)$ \\
\hline Chest pain & $3(25.0)$ \\
\hline Lymphadenopathy & $3(25.0)$ \\
\hline $\begin{array}{l}\text { Other: haemoptysis, erythema nodosum, } \\
\text { ganglion, hoarseness and back pain }\end{array}$ & 1 (8.3) each \\
\hline \multicolumn{2}{|l|}{ Diagnostic investigations ${ }^{*}$} \\
\hline Chest X-ray & $10(83.3)$ \\
\hline Computed tomography scan of chest & $6(50.0)$ \\
\hline Sputum culture and sensitivity testing & $5(41.7)$ \\
\hline $\begin{array}{l}\text { Polymerase chain reaction-based test, e.g. } \\
\text { GeneXpert }\end{array}$ & $5(41.7)$ \\
\hline Sputum microscopy & $5(41.7)$ \\
\hline Tissue biopsy (e.g. pleura, lymph node) & $5(41.7)$ \\
\hline Bronchoscopy & $3(25.0)$ \\
\hline Pleural fluid aspiration & $3(25.0)$ \\
\hline Fine-needle aspiration biopsy & $1(8.3)$ \\
\hline Computed tomography abdomen & $1(8.3)$ \\
\hline Magnetic resonance imaging: spine & $1(8.3)$ \\
\hline Interferon gamma release assays & $0(0)$ \\
\hline
\end{tabular}

and fibromyalgia $(n=1)$. One participant was started empirically on a DS-TB regimen without sending pleural fluid for TB culture, which delayed the diagnosis of DR-TB.

Participants collected their medication from government clinics $(n=7)$, private pharmacies $(n=7)$ and training hospitals $(n=2)$ and 
also reported switching between the above options. Half of the participants received directly observed treatment, but found it inconvenient due to high transport costs and missed academic time:

'I got to campus health and asked, "I want to get my medication here," and they told me, "No we don't do that." I asked where students get their medication and the sister said, "You have to go to a registered TB clinic". I said, "Sister but I don't have a car. When am I going to go? I have classes." ... Then I went to occupational health and [the sister working there] said "I can't do anything for you. The university can't follow you up and we give you medication." It was just such a grey area, it was such a struggle ... Eventually sister said, "You know what, this is nonsense, I'm going to take you," and she started giving me the medication. It should never have been that hard to get medication. (P1)

Several participants highlighted the need for comprehensive student occupational health services:

'We need someone responsible for occupational health for students. You have students in the hospital doing the same things that the doctors are doing and the doctors have occupational health - why wouldn't the students have that as well? There is a huge disconnect [and] misunderstanding.' (P11)

\section{Impact of disease}

Eight participants experienced side-effects of treatment, most commonly gastrointestinal side-effects $(n=8)$, difficulty concentrating $(n=3)$, as well as more serious adverse events including hepatotoxicity $(n=2)$, peripheral neuropathy $(n=2)$ and depression $(n=2)$.

Participants with DR-TB experienced an increased number and severity of side-effects and found treatment very difficult to endure, with one participant strongly considering discontinuing treatment:

'Taking the pills literally takes a few minutes, but for me, the whole day I think about taking the pills then every hour I'll be counting down till I take the pills and then the minutes and seconds. Then I sit there with my pills and then I take my pills and then I sit there in fear of the side-effects. I feel nauseous, I have diarrhoea, not able to sleep, still thinking, "Oh my gosh, I have to do this again tomorrow." The pain from the injection consumes your whole day.' (P2)

Three participants had side-effects that resulted in long-term disability including hearing loss, depression and decreased visual acuity:

'My hearing loss has been a very difficult adjustment and I am often seen as not paying attention or not knowing answers, when in fact [the problem is] I cannot hear normally.' (P3)

Participants missed academic time due to the initial 2-week isolation period, clinic visits to collect treatment and follow-up appointments. Three students had to interrupt their studies, while others were under severe pressure to catch up missed clinical time in order for them to graduate with their year group. Participants reported substantial expenses, including specialist appointments, investigations, treatment costs (DS-TB: ZAR1 060 for 6 months) and transport costs to the clinics (ZAR700 for 6 months). Participants also incurred costs as a result of treatment complications (hearing aids) and complications due to TB disease (laparoscopic repair of inguinal hernia after chronic cough). Few students had comprehensive medical aid cover. Other costs included paying the tuition fees and accommodation of the additional years of studying. Participants' average estimated personal expenses for DS-TB were ZAR25 000 (to a maximum of ZAR80 000) and for DR-TB up to ZAR104 000.

Three participants mentioned that their experience with TB had made them realise how difficult it can be to undergo treatment. Most participants felt that TB had increased their empathy toward patients and motivated them to provide patient-centred care:

'There's a certain understanding that comes with an experience like this that can't be taught. I understand now how important it is to treat the whole patient and not just the disease because illness is far reaching and infiltrates every aspect of a person's life.' (P11)

For some participants, their personal experience created a desire to help to make a difference to the TB burden. Several participants noted that their experience made them realise what risks their occupation poses:

'I didn't realise that when I went into medicine that I was exposing myself to people's diseases - in fact all the diseases came running towards us. Being a doctor and having the profession is not worth me being so close to death.' (P2)

Two participants, both of whom had DR-TB, wanted to leave medical school:

'I came back at the insistence of my family because I had completed so much of my degree. I had to receive psychological counselling to help me cope with the adjustment and post-traumatic stress. I do not see myself being a doctor involved in the clinical care of patients. Once I complete my degree and internship I don't think I will continue a career in medicine.' (P3)

\section{Support}

Two participants struggled to access any family support, as their families lived in other provinces; some relied heavily on support from friends at university. The majority of participants $(n=7)$ strongly expressed their disappointment with the support that they received from their universities:

'My university protocol was dismal to be honest. I wasn't sure what to do or where to go. It was just another stressor I didn't need when I was trying to get better.' (P11)

'I think faculties really need to know this is their problem, whether they want to admit it or not. They need to do more about it.' (P2)

\section{TB infection control in training hospitals}

Most participants $(n=10)$ cited poor TB-IC at training hospitals as their major risk factor for occupational TB. The administrative controls (fast-tracking patients with $\mathrm{TB}$ symptoms and isolating smear-positive patients) were poorly implemented and environmental controls (opening windows, mechanical ventilation) were infrequently used.

'Some windows are bolted shut. Others are never opened because nobody [wants to] get cold in winter.' (P2)

Often the only TB-IC method available to students was the N95 respirator (the lowest tier of protection on the Centers for Disease Control and Prevention hierarchy of TB-IC). Both universities instituted N95 fit-testing programmes in 2013, and supply respirators to students annually, although problems with access to respirators persist:

'Respirators are tough: they are not nice to wear, they are hot and stuffy, and the patients can't understand you, especially if there is a language barrier. So you often have to take it off and repeat yourself for them to hear you, which actually defeats the point.' (P10)

There is also a lack of an institutional safety culture, with poor TB-IC implementation and few positive senior clinician role models:

"We had our masks, we take them out and put them on and sometimes the doctors go, "Oh look at the third years!" [It made 
us wonder] are they not aware, do they know something that we don't? It is very confusing.' (P11)

Participants felt the high-risk zones for TB exposure were areas where undifferentiated patients were seen: medical, surgical and trauma emergency units and peripheral training hospitals and clinics:

'F1 [the medical emergency unit] is a mess; it is scary. It really is scary.' (P11)

Returning to this clinical environment after developing occupational TB disease left most participants feeling scared and concerned about TB reinfection:

'I saw the first patient coughing and they told me the patient had TB; I felt like I was having an anxiety attack. I just had to leave the room, I didn't want to see a patient, I didn't want to be around a patient, I just wanted to leave.' (P10)

Participants made several recommendations to improve diagnosis, treatment and psychosocial support for students diagnosed with TB (Table 2).

\section{Discussion}

This study highlights the vulnerability of medical students at two of the eight SA medical schools, documenting substantial TB exposure and risk of progression to occupational TB. In comparison with data from Naidoo et al. ${ }^{[11]}$ reporting TB disease in medical doctors in KwaZulu-Natal, medical students had a greater incidence of extrapulmonary TB (50\% v. 20\%) and higher incidence of DR-TB (16.7\% v. $10 \%)$. Fewer students than doctors ( $25 \%$ v. $75 \%)$ reported having easy access to specialised diagnostic procedures. Students (41.7\%) had a lower percentage of initial correct diagnosis compared with doctors (52.5\%), and experienced longer diagnostic delays. This may reflect the lack of student occupational health services and the perceived lower risk for occupational TB among students. Students consistently indicated that they struggled to access diagnostics and treatment, with prominent opportunity costs (lost academic time during treatment collection) and direct costs for transport.

Participants with DR-TB suffered long-term disability due to treatment side effects that impacted their ability to continue with clinical medicine, highlighting the urgent need for less toxic DR-TB treatment regimens. This correlated with themes identified by Padayatchi et al. ${ }^{[12]}$ (prolonged morbidity, attrition from the field, psychological impact, weak support structures and poor infection control). Despite severe psychosocial impacts, most participants showed astounding resilience, drawing parallels between their experiences of $\mathrm{TB}$ and those of the patients they care for. The majority indicated that their TB diagnosis had given them new insight into the impact of disease and treatment in their patients' lives, corroborating Woolf's findings. ${ }^{[14]}$
Working in a healthcare system where TB-IC is poorly implemented, participants perceived their risk of $\mathrm{TB}$ reinfection to be very high. Negative clinician role modelling was found to play an important role in discouraging respirator usage, as reported in previous studies in this setting. ${ }^{[6]}$

As stipulated under the Regulations for Biological Hazardous Agents and the Occupational Health and Safety Act No. 85 of 1993, healthcare workers are legally entitled to reasonable protection from TB exposure through TB-IC measures, annual TB screening and access to diagnostic and treatment services. ${ }^{[15]}$ For health science students, however, there is uncertainty as to who should offer these services (the universities or the hospitals where clinical training is based), and whether students are protected by the Compensation for Occupational Injuries and Diseases Act No. 130 of 1993.

The lack of national guidance and policies has led to an unjust situation where SA health science students train in a hazardous working environment with no recourse to assistance with diagnostic and treatment costs for an occupational disease. There is an urgent need for SA medical training institutions and the National Department of Health to develop policies for the provision and funding of health services for students with occupationally acquired TB disease.

The findings of this study could likely be extrapolated to medical students at other SA universities and other high-TB-burden countries. Other vulnerable groups highly exposed to TB disease due to poor TB-IC in healthcare settings include nursing students, allied health students, and non-clinical staff, for example porters, working in clinical areas. Further research is needed to quantify the burden of occupational TB in these subpopulations with tailored occupational health policies offering comprehensive support.

\section{Study limitations}

This study utilised self-reporting to identify eligible participants, as institutional data on occupational TB disease in medical students are not routinely collected. This study did not include participants who developed latent $\mathrm{TB}$ during their training and developed $\mathrm{TB}$ disease after graduation. TB is highly stigmatised, which also impacts willingness to disclose it. Given these factors, it is likely that this study underrepresents the true burden of TB disease in medical students at the two universities.

\section{Conclusion}

Medical students training in TB-endemic settings are at high risk of occupationally acquired TB. Students experienced significant diagnostic delays and barriers to accessing TB care. Participants with DR-TB interrupted their studies, incurred large expenses and experienced severe side-effects. Although the experience of TB disease increased students' empathy with patients, they reported feeling greater vulnerability upon returning to the clinical

Table 2. Recommendations by participants to address TB in medical students

1. Strengthen TB-IC in all healthcare facilities, from clinics, district hospitals to tertiary hospitals.

2. Develop and implement TB-prevention policies at all healthcare training institutions.

3. Ensure availability of fit-tested $\mathrm{N} 95$ respirators at all times, free of charge to students.

4. Provide comprehensive student occupational health services, including screening, diagnostic investigations and campus-based collection of treatment, free of charge.

5. Provide support structures for students who develop TB, including: alternative accommodation arrangements for students during the isolation period, campus-based support groups of students who have developed TB, monthly check-ups to provide treatment support and additional academic support. 
environment. Students reported poor implementation of TB-IC in their training institutions as their major risk factor for occupational disease. Comprehensive occupational health services and institutional policies for TB prevention among health science students are urgently needed. Further research to quantify the burden of occupational TB in medical students and other neglected subpopulations should be prioritised.

Acknowledgements. We thank all participants for sharing their stories and are grateful for the assistance and insight provided by Zolelwa Sifumba. We thank Dr Donald Skinner for advice on qualitative data analysis and Dr Moleen Zunza for assistance with the statistical analysis. We thank Capital for Good (Geneva Global) for proving funding for interview transcriptions and the lucky-draw prize through the non-governmental organisation TB Proof.

. World Health Organization. Global Tuberculosis Report 2016. Geneva: WHO Press, 2016. http://www. who.int/tb/publications/global_report/en/(accessed 27 February 2017).

2. Menzies D, Joshi R, Pai M. Risk of tuberculosis infection and disease associated with work in healthcare settings. Int J Tuberc Lung Dis 2007;11(6):593-605.

3. Joshi R, Reingold AL, Menzies D, Pai M. Tuberculosis among healthcare workers in low- and middleincome countries: A systematic review. PLoS Med 2006;3(12):e494. https://doi.org/10.1371/journal. pmed.0030494

4. O'Donnell MR, Jarand J, Loveday M, et al. High incidence of hospital admissions with multidrugresistant and extensively drug-resistant tuberculosis among South African healthcare workers. Ann
(n) Intern Med 2010;153(8):516-522. https://doi.org/10.1059/0003-4819-153-8-201010190-00008
5. Health Systems Trust. The National Healthcare Facilities Baseline Audit, 2012. https://www.health-e. 6. 7 . wp -content/uploads/2013/09/National-Health-Facilites-Audit pdf (accessed 27 February 2017). 6. Van der Westhuizen $\mathrm{H}$ Kotze $\mathrm{J}$ Narotam $\mathrm{H}$, von Delft A, Willems B, Dramowski A. Knowed 6. Nan der Westhizente Kotze J, Narotan H, von Delf $\mathrm{A}$, Willems B, Dramowski A. Knowledge attitudes and practices regarding TB infection among health science students in a TB-

7. McCarthy KM, Scott LE, Gous N, et al. High incidence of latent tuberculous infection among McCarthy KM, Scott LE, Gous N, et al. High incidence of latent tuberculous infection among
South African health workers: An urgent call for action. Int I Tuberc Lung Dis 2015;19(6):647653. https://doi.org/10.5588/ijtld.14.0759

653. https://doi.org/10.5588/ijtld.14.0759
8. Baussano I, Nunn P, Williams B, Pivetta E, Bugiani M, Scano F. Tuberculosis among healthcare Baussano I, Nunn P, Williams B, Pivetta E, Bugiani M, Scano F. Tuberculosis among
workers. Emerg Infect Dis 2011;17(3):488-494. https://doi.org/10.3201/eid1703.100947

workers. Emerg Infect Dis 2011;17(3):488-494. https://doi.org/10.3201/eid1703.100947 9. Van Rie A, McCarthy K, Scott L, Dow A, Venter WDF, Stevens WS. Prevalence, risk factors and risk perception of tuberculosis infection among medical students and healthcare workers
in Johannesburg, South Africa. S Afr Med J 2013;103(11):853-857. http://doi.org/10.7196/ SAMJ.7092

10. Dramowski A, Marais F, Willems B, Mehtar S. Does undergraduate teaching of Infection Prevention and Control (IPC) adequately equip medical graduates for clinical practice? Afr J Health Professions Educ 2015;7(1Suppl1):S105-S110. https://doi.org/10.7196/AJHPE.500

11. Naidoo A, Naidoo S, Gathiram P, Lalloo U. Tuberculosis in medical doctors - a study of personal experiences and attitudes. S Afr Med J 2013:103(3):176-180. https://doi.org/10.7196/SAMJ.6266

1. Padaychi $N$ Daftary A, Moodley T, Madasein R, Ramjec A. Case series of the lon-term Pacher long-term psychosocial impact of drug-resista Lung Dis 2010;14(8):960-966.

3. Von Delft A, Drawski A, Sifumba Z, et al. Exposed, but not protected: More is needed to prevent drug-resistant tuberculosis in healthcare workers and students. Clin Infect Dis 2016;62(Suppl3):S275-S280. https://doi.org/10.1093/cid/ciw037

14. Woolf K, Cave J, McManus IC, Dacre JE. 'It gives you an understanding you can't get from an book.' The relationship between medical students' and doctors' personal illness experiences an their performance: A qualitative and quantitative study. BMC Med Educ 2007;7:50. https://doi org/10.1186/1472-6920-7-50

15. Zungu M, Malotle M. Do we know enough to prevent occupationally acquired tuberculosis in healthcare workers ? Occup Health South Africa 2011;7(5):17-21.

Accepted 27 February 2017. 